\begin{tabular}{|c|l|}
\hline Title & Simple improvements to classical bubble nucleation models \\
\hline Author(s) & Tanaka, Kyoko K.; Tanaka, Hidekazu; A ngelil, Raymond; Diemand, Juerg \\
\hline Citation & $\begin{array}{l}\text { Physical review E, 92(2), 22401 } \\
\text { https://doi.org/L0.1103/PhySRevE.92.022401 }\end{array}$ \\
\hline Issue Date & 2015-08-03 \\
\hline Doc URL & http://hdl.handle.net/2115/59820 \\
\hline Rights & @2015A merican Physical Society \\
\hline Type & article \\
\hline File Information & PhysRevE.92.022401.pdf \\
\hline
\end{tabular}

Instructions for use 


\title{
Simple improvements to classical bubble nucleation models
}

\author{
Kyoko K. Tanaka and Hidekazu Tanaka \\ Institute of Low Temperature Science, Hokkaido University, Sapporo 060-0819, Japan
}

Raymond Angélil and Jürg Diemand

Institute for Computational Science, University of Zürich, 8057 Zürich, Switzerland

(Received 8 October 2014; revised manuscript received 12 June 2015; published 3 August 2015)

\begin{abstract}
We revisit classical nucleation theory (CNT) for the homogeneous bubble nucleation rate and improve the classical formula using a correct prefactor in the nucleation rate. Most of the previous theoretical studies have used the constant prefactor determined by the bubble growth due to the evaporation process from the bubble surface. However, the growth of bubbles is also regulated by the thermal conduction, the viscosity, and the inertia of liquid motion. These effects can decrease the prefactor significantly, especially when the liquid pressure is much smaller than the equilibrium one. The deviation in the nucleation rate between the improved formula and the CNT can be as large as several orders of magnitude. Our improved, accurate prefactor and recent advances in molecular dynamics simulations and laboratory experiments for argon bubble nucleation enable us to precisely constrain the free energy barrier for bubble nucleation. Assuming the correction to the CNT free energy is of the functional form suggested by Tolman, the precise evaluations of the free energy barriers suggest the Tolman length is $\simeq 0.3 \sigma$ independently of the temperature for argon bubble nucleation, where $\sigma$ is the unit length of the Lennard-Jones potential. With this Tolman correction and our prefactor one gets accurate bubble nucleation rate predictions in the parameter range probed by current experiments and molecular dynamics simulations.
\end{abstract}

DOI: 10.1103/PhysRevE.92.022401 PACS number(s): 64.60.Q-, 64.70.fh, 64.70.fm, 68.03.Cd

\section{INTRODUCTION}

Bubble nucleation in liquid is a liquid-to-vapor transition [1] phenomenon, and plays an important role in many areas of science and technology, e.g., degassification of steel [1], bubble jet printers [2], volcanism [3-5], the direct detection of dark matter [6-9], and medicine [10,11]. Bubble nucleation occurs in a metastable liquid under a pressure below its equilibrium vapor pressure. The liquid phase can even exist at negative pressures, and bubble nucleation is often observed at negative pressure, although there are no fundamental differences between a boiling, superheated (positive pressure) and a cavitating, stretched (negative pressure) liquid [12]. Studies of homogeneous liquid-vapor nucleation typically use the classical nucleation theory (CNT) for the bubble nucleation rate. However, the range of applicability of the CNT is not well understood.

Numerical techniques such as molecular dynamics and Monte Carlo simulations are powerful methods which can resolve details of the nucleation process and provide useful test cases for nucleation models [13-28]. Typically, these simulations show large deviations from the CNT predictions. The CNT nucleation rates usually underestimate the bubble nucleation rates by very large factors [15-18,20-22,25,26]. Most of the simulations for bubble nucleation in the literature use around $10^{5}$ or fewer atoms, making it difficult to measure nucleation rates directly.

Recently, Diemand et al. [26] presented large-scale, microcanonical molecular dynamics simulations of homogeneous bubble nucleation with $5 \times 10^{8}$ Lennard-Jones atoms, and succeeded in measuring nucleation rates in the range $10^{21}-10^{25} \mathrm{~cm}^{-3} \mathrm{~s}^{-1}$ for argon by directly resolving bubble nucleation events in the steady state nucleation phase. The measured rates agree well with the CNT within two orders of magnitude in the superheated boiling regime (positive ambient pressure), while the CNT prediction underestimates the nucleation rates significantly in the cavitation regime (lower temperatures and negative pressures).

The kinetics of explosive cavitation in liquid has also been investigated in laboratory experiments, which have measured the superheat temperature for liquid argon at both positive and negative pressures, by pulse heating liquid around a thin wire in a negative pressure wave [12,29]. Using this method, Vinogradov et al. [12] measured nucleation rates of $10^{16}-10^{18} \mathrm{~cm}^{-3} \mathrm{~s}^{-1}$ in superheated liquid argon of high purity.

The recent advances in molecular dynamics simulations and experiments enable us to precisely test theoretical bubble nucleation models and also to improve them. One of the most serious problems in the CNT model is that the bulk value of the surface tension is used to evaluate nanobubble formation energy. Since the nucleation rate of the CNT depends exponentially on the formation energy, an incorrect estimate of it can cause a huge error in the nucleation rate. According to Tolman's correction [30], the surface tension at surfaces of small nuclei (bubbles or droplets) is dependent on their radius. A model parameter called the Tolman length can be determined by the measurement of the surface tension of small nuclei [26,31-40]. Diemand et al. [26] showed that the CNT model agrees well with the nucleation rate from their MD simulations, by using such a model for the surface tension with a proper Tolman length. The introduction of a Tolman correction can also significantly improve model predictions in vapor-to-liquid droplet nucleation [40].

In bubble nucleation, additional detail in the treatment of the process is required, in comparison to droplet nucleation, because of the variable vapor pressure and density in bubbles as they grow [41-46]. The vapor pressure in bubbles varies as they grow and has a significant effect on their growth rates and on the pre-exponential factor in the CNT formula for the 
nucleation rate. Thus we have to solve the two-dimensional evolution (i.e., the radius and pressure) for the bubble growth process. An extensive study of this problem has already been done by Kagan [41]. Kagan showed that the pre-exponential factor is strongly dependent on the ambient liquid pressure reduced from the saturation. Despite this, a constant prefactor is usually adopted in the widely used CNT. Furthermore, the formation energy of a bubble also depends on the vapor pressure. Although the vapor pressure is approximately given by the saturated pressure for critical bubbles, a more accurate vapor pressure is necessary for the evaluation of the formation energy in the CNT [1].

Although these treatments have been developed in individual studies, they are not included directly in the widely used CNT. For comparisons with recent molecular simulations and laboratory experiments, we should use a precisely crafted expression for the CNT bubble nucleation rate. Detailed comparisons also enable us to correctly determine the Tolman length in the model of the surface tension.

In this paper, we first present a more complete expression for the nucleation rate, by summarizing the above studies (Sec. II). Next, we compare the improved model with the original CNT or with the measured values in the recent MD simulations and experiments (Sec. III). We find that the difference between the improved model and the CNT could be several orders of magnitude for realistic bubble nucleation parameters. Comparisons with MD simulations can determine the Tolman length more accurately thanks to the more accurate prefactor in our model. From our comparisons at various temperatures, the Tolman length is obtained as $\simeq 0.3 \sigma$, where $\sigma$ is the unit length of the Lennard-Jones potential. A summary of our findings can be found in Sec. IV.

\section{NUCLEATION THEORY}

\section{A. Classical expression}

The bubble nucleation rate is the number of stable bubbles formed per unit time per unit volume and is given by $[1,47]$

$$
J=J_{0} n_{\mathrm{e}}\left(i_{\mathrm{c}}\right),
$$

where $i_{\mathrm{c}}$ is the number of gaseous molecules in a critical bubble, $J_{0}$ is the prefactor in the nucleation rate, and the number density of bubbles $n_{\mathrm{e}}(i)$ is given by

$$
n_{\mathrm{e}}(i)=n_{0} \exp \left(-\frac{\Delta G(i)}{k T}\right)
$$

where $T$ is the temperature, $k$ is the Boltzmann constant, $\Delta G(i)$ is the minimum work for the formation of a bubble with $i$ molecules, and $n_{0}$ is the number density of liquid molecules.

In the classical nucleation theory (CNT), the minimum work for the formation of the critical bubble is

$$
\Delta G_{\mathrm{CNT}}=\frac{16 \pi \gamma^{3}}{3\left(P_{\mathrm{eq}}-P_{1}\right)^{2}},
$$

where $\gamma$ is the surface tension, and $P_{\text {eq }}$ and $P_{1}$ are the equilibrium vapor pressure at saturation and the liquid pressure, respectively. The prefactor $J_{0}$ is given in the CNT by

$$
J_{0, \mathrm{CNT}}=\sqrt{\frac{2 \gamma}{\pi m}}
$$

which is the same as in the droplet nucleation. In the above, $m$ is the molecular mass. Thus, the widely used expression of the CNT nucleation rate is

$$
J_{\mathrm{CNT}}=\sqrt{\frac{2 \gamma}{\pi m}} n_{0} \exp \left[-\frac{16 \pi \gamma^{3}}{3 k T\left(P_{\mathrm{eq}}-P_{1}\right)^{2}}\right] .
$$

We will describe more accurate expressions for $\Delta G$ and $J_{0}$ in Secs. II B and II C.

\section{B. Free energy for bubble formation and the Poynting correction}

The minimum work $\Delta G$ for the formation of a bubble with radius $r$ is given by $[1,47]$

$$
\Delta G=\frac{4 \pi r^{3}}{3}\left\{\frac{1}{v_{\mathrm{g}}}\left[\mu_{\mathrm{g}}\left(P_{\mathrm{g}}\right)-\mu_{\mathrm{l}}\left(P_{1}\right)\right]-\left(P_{\mathrm{g}}-P_{1}\right)\right\}+4 \pi r^{2} \gamma
$$

$\mu_{\mathrm{g}}$ and $\mu_{1}$ are the chemical potentials of the gas and liquid respectively, $P_{\mathrm{g}}$ is the gaseous pressure in the bubble, and the molar volume of a gas $v_{\mathrm{g}}$ is given by $k T / P_{\mathrm{g}}$, assuming the ideal gas. Equation (6) also assumes spherical bubbles. Note that the work for bubble formation is a function of two variables $\left(r, P_{\mathrm{g}}\right)$ or $(r, i)$. The number of molecules in the bubble is given by

$$
i=\frac{4 \pi r^{3} P_{\mathrm{g}}}{3 k T} \text {. }
$$

Previous studies have investigated the minimum work for a bubble formation with two variables, taking into account bubble compressibility [41-46]. Here we use the two variables $r$ and $P_{\mathrm{g}}$.

The work $\Delta G$ has a maximum value along the path of the bubble growth. Such a maximum point on the path corresponds to the critical bubble and is given by the saddle point in the two-dimensional plane $\left(r, P_{\mathrm{g}}\right)$ [11]. In the next subsection, we describe the growing path in the vicinity of the saddle point. The radius and the internal pressure of the critical bubble are thus obtained from the conditions

$$
\left(\frac{\partial \Delta G}{\partial P_{\mathrm{g}}}\right)_{r}=\left(\frac{\partial \Delta G}{\partial r}\right)_{P_{\mathrm{g}}}=0
$$

Since the first-order derivatives of the minimum work are determined by

$$
\left(\frac{\partial \Delta G}{\partial r}\right)_{P_{\mathrm{g}}}=4 \pi r^{2}\left[\frac{P_{\mathrm{g}}}{k T}\left[\mu_{\mathrm{g}}\left(P_{\mathrm{g}}\right)-\mu_{\mathrm{l}}\right]-\left(P_{\mathrm{g}}-P_{1}-\frac{2 \gamma}{r}\right)\right]
$$

and

$$
\left(\frac{\partial \Delta G}{\partial P_{\mathrm{g}}}\right)_{r}=\frac{4 \pi r^{3}}{3}\left[\mu_{\mathrm{g}}\left(P_{\mathrm{g}}\right)-\mu_{1}\right]
$$

the critical size of the bubble $r_{\mathrm{c}}$ and the critical gas pressure $P_{\mathrm{g}, \mathrm{c}}$ are determined by

$$
\begin{aligned}
& \mu_{\mathrm{g}}\left(P_{\mathrm{g}, \mathrm{c}}\right)=\mu_{1}, \\
& r_{c}=\frac{2 \gamma}{P_{\mathrm{g}, \mathrm{c}}-P_{1}} .
\end{aligned}
$$


Equations (11) and (12) indicate the chemical equilibrium and the mechanical equilibrium at the saddle point, respectively. Using Eqs. (11) and (12), the minimum work for the formation of the critical bubble is

$$
\Delta G\left(r_{c}, P_{\mathrm{g}, \mathrm{c}}\right)=\frac{4 \pi r_{c}^{2} \gamma}{3} .
$$

Using Eqs. (9)-(12), the second-order derivatives of the minimum work at the saddle point are given by

$$
\begin{gathered}
\left(\frac{\partial^{2} \Delta G}{\partial r^{2}}\right)_{P_{\mathrm{g}}}=-8 \pi \gamma<0, \\
\left(\frac{\partial^{2} \Delta G}{\partial P_{\mathrm{g}}^{2}}\right)_{r}=\frac{4 \pi r_{\mathrm{c}}^{3}}{3 P_{\mathrm{g}, \mathrm{c}}}>0,
\end{gathered}
$$

and

$$
\left(\frac{\partial^{2} \Delta G}{\partial r \partial P_{\mathrm{g}}}\right)=0
$$

With Eqs. (13)-(16), the work for bubble formation around the critical size is given by [48]

$$
\Delta G=\frac{4 \pi r_{c}^{2} \gamma}{3}-4 \pi \gamma\left(r-r_{c}\right)^{2}+\frac{2 \pi r^{3}}{3 P_{\mathrm{g}, \mathrm{c}}}\left(P_{\mathrm{g}}-P_{\mathrm{g}, \mathrm{c}}\right)^{2}
$$

up to second order accuracy in $\left(r-r_{c}\right)$ and $\left(P_{\mathrm{g}}-P_{\mathrm{g}, \mathrm{c}}\right)$. This expression shows that the point $\left(r_{\mathrm{c}}, P_{\mathrm{g}, \mathrm{c}}\right)$ is indeed the saddle point.

The gas pressure $P_{\mathrm{g}, \mathrm{c}}$ in the critical bubble is determined by Eq. (11). Integrating $d \mu=v d P$, we obtain

$$
\mu_{\mathrm{g}}\left(P_{\mathrm{g}, \mathrm{c}}\right)-\mu_{\mathrm{g}}\left(P_{\mathrm{eq}}\right)=k T \ln \left(\frac{P_{\mathrm{g}, \mathrm{c}}}{P_{\mathrm{eq}}}\right)
$$

for gas and

$$
\mu_{1}\left(P_{1}\right)-\mu_{1}\left(P_{\text {eq }}\right)=v_{1}\left(P_{1}-P_{\text {eq }}\right)
$$

for liquid. In Eq. (18) we used the equation of state for ideal gas, $v_{g}=k T / P_{g}$, and $v_{l}$ is assumed to be constant in Eq. (19). Noting $\mu_{g}\left(P_{\mathrm{eq}}\right)=\mu_{l}\left(P_{\text {eq }}\right)$, Eqs. (11), (18), and (19) yields

$$
\ln \left(\frac{P_{\mathrm{g}, \mathrm{c}}}{P_{\mathrm{eq}}}\right)=\frac{v_{1}}{v_{\mathrm{eq}}}\left(\frac{P_{1}}{P_{\mathrm{eq}}}-1\right) .
$$

In (20), $v_{\mathrm{eq}}\left(=k T / P_{\mathrm{eq}}\right)$ is the molecular volume of ideal gas at the equilibrium pressure.

In the classical theory, the right hand side of Eq. (20) is set to be zero because the ratio $v_{\mathrm{l}} / v_{\mathrm{g}}$ is small. This approximation in Eq. (20) gives $P_{\mathrm{g}, \mathrm{c}}=P_{\mathrm{eq}}$. Then, in the CNT, the critical radius is given by

$$
r_{\mathrm{c}, \mathrm{CNT}}=\frac{2 \gamma}{P_{\mathrm{eq}}-P_{1}} .
$$

Blander and Katz [1] keep the small term proportional to $v_{1} / v_{\text {eq }}$ in Eq. (20) and derive more accurate expressions for the critical radius and the nucleation rate than CNT. They termed this correction to CNT as the Poynting correction. Here we label this correction as PCNT. In PCNT, the gaseous pressure in the bubble is obtained as

$$
\begin{aligned}
\frac{P_{\mathrm{g}, \mathrm{c}}}{P_{\mathrm{eq}}} & =\exp \left(\frac{v_{1}}{v_{\mathrm{eq}}} \frac{P_{1}-P_{\mathrm{eq}}}{P_{\mathrm{eq}}}\right) \\
& \simeq 1+\frac{v_{1}}{v_{\mathrm{eq}}}\left(\frac{P_{1}-P_{\mathrm{eq}}}{P_{\mathrm{eq}}}\right)+\frac{1}{2}\left(\frac{v_{1}}{v_{\mathrm{eq}}} \frac{P_{1}-P_{\mathrm{eq}}}{P_{\mathrm{eq}}}\right)^{2},
\end{aligned}
$$

then we have

$$
P_{\mathrm{g}, \mathrm{c}}-P_{1}=\left(P_{\mathrm{eq}}-P_{1}\right) \delta,
$$

where $\delta$ is the Poynting correction factor given by

$$
\delta=\left[1-\frac{v_{1}}{v_{\mathrm{eq}}}+\frac{P_{\mathrm{eq}}-P_{1}}{2 P_{\mathrm{eq}}}\left(\frac{v_{1}}{v_{\mathrm{eq}}}\right)^{2}\right] .
$$

The expansion in Eq. (22) is valid even if $P_{1}$ is far from $P_{\text {eq }}$ because of the factor $v_{1} / v_{\mathrm{eq}}$ is small $(\sim 0.01-0.1)$. This indicates that $P_{\mathrm{g}}\left(r_{\mathrm{c}}\right)$ is close to $P_{\text {eq }}$ even if $P_{\mathrm{l}}$ is far from $P_{\mathrm{eq}}$. But, the gaseous pressure $P_{\mathrm{g}}$ can deviate considerably from $P_{\mathrm{eq}}(r)$ when $r$ is far from $r_{\mathrm{c}}$. We find that in the Poynting correction factor derived by Blander and Katz, the second order term of the small ratio $v_{1} / v_{\text {eq }}$ is incorrect.

Note that the approximation of ideal gas is inaccurate at a high temperature where the equilibrium pressure $P_{\text {eq }}(T)$ is considerably large. We also derive the the Poynting correction factor for such a nonideal case with the first order accuracy. Since $P_{\mathrm{g}, \mathrm{c}}$ is close to $P_{\text {eq }}$ for critical bubbles, $v_{\mathrm{gas}}$ can be replaced by $v_{\text {eq }}\left[=v_{\text {gas }}\left(P_{\text {eq }}\right)\right]$ in the first order approximation and we obtain instead of Eq. (18)

$$
\mu_{\mathrm{g}}\left(P_{\mathrm{g}, \mathrm{c}}\right)-\mu_{\mathrm{g}}\left(P_{\mathrm{eq}}\right)=v_{\mathrm{eq}}\left(P_{\mathrm{g}, \mathrm{c}}-P_{\mathrm{eq}}\right) .
$$

As for $v_{\text {eq }}$, we include the nonideal effect. Using the second virial coefficient $B_{2}$, the molecular volume $v_{\text {eq }}$ of nonideal gas is given by [47]

$$
v_{\mathrm{eq}}=k T / P_{\mathrm{eq}}+B_{2}(T) \text {. }
$$

Although we use Eq. (25) instead of (18), we obtain the same $P_{\mathrm{g}, \mathrm{c}}$ as Eq. (22) up to the first order term and thus have $\delta=$ $1-v_{\mathrm{l}} / v_{\mathrm{eq}}$. Hence, by using $v_{\mathrm{eq}}$ of Eq. (26) in Eq. (23), we can obtain the Poynting correction factor for nonideal gas with first order accuracy.

The critical radius in the PCNT is obtained as

$$
r_{\mathrm{c}, \mathrm{PCNT}}=\frac{2 \gamma}{\left(P_{\mathrm{eq}}-P_{1}\right) \delta} .
$$

With this Poynting correction for $r_{\mathrm{c}}$, the nucleation rate is given by

$$
J=J_{0} n_{0} \exp \left[-\frac{16 \pi \gamma^{3}}{3 k T\left(P_{\mathrm{eq}}-P_{1}\right)^{2} \delta^{2}}\right] .
$$

We will consider the prefactor $J_{0}$ in the next subsection. Although $1-\delta\left(\simeq v_{\mathrm{l}} / v_{\mathrm{g}}\right)$ is usually small, the difference in the nucleation rate between the CNT and PCNT can be large because of the strong exponential dependence. This correction is necessary especially at a relatively high temperature where the ratio $v_{1} / v_{\mathrm{g}}$ is not so small due to high $P_{\text {eq }}$. At such a high saturated pressure, it is also necessary to include the nonideal gas effect for $v_{\text {eq }}$ [Eq. (26)].

For a more accurate evaluation of the nucleation rate, we also need to take into account the deviation of the surface 

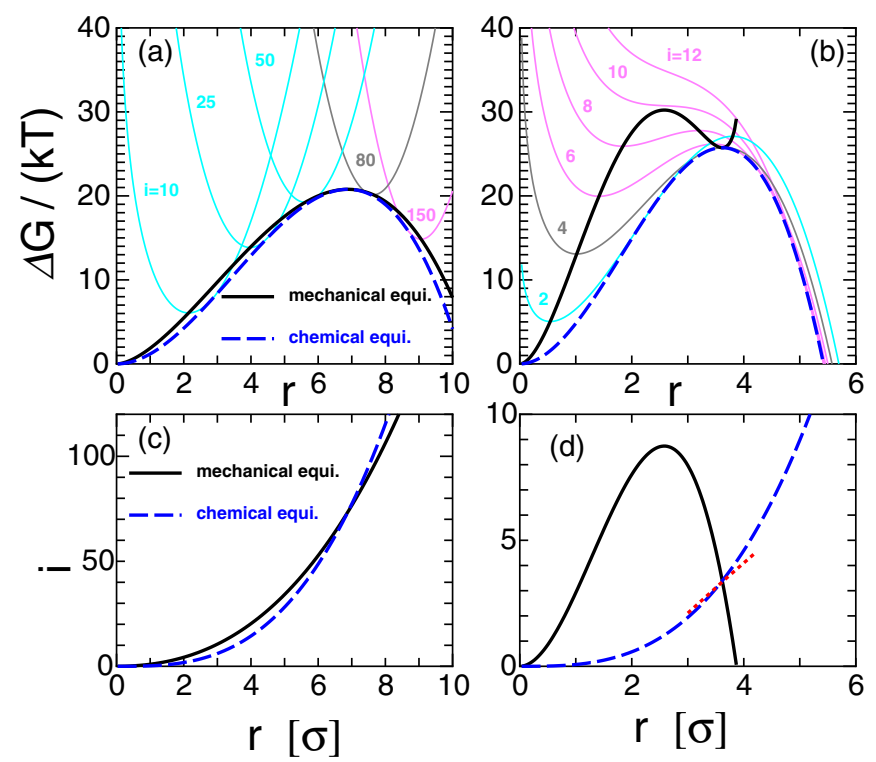

FIG. 1. (Color online) Free energy for bubble formation $\Delta G$ as a function of the bubble radius for Lennard-Jones system with $T^{*}=0.855$ (a) and $0.7(\mathrm{~b})$. The liquid pressures are $P_{l}=0.017 \varepsilon \sigma^{-3}$ and $P_{l}=-0.16 \varepsilon \sigma^{-3}$ for $T^{*}=0.855$ and 0.7 , respectively (see text for details). The black solid curves assume the mechanical equilibrium, while the blue dashed indicate the chemical equilibrium. The thin solid curves show $\Delta G$ for various constant bubble molecule numbers $i$. Panels (c) and (d) show the relations between $i$ and $r$ in the mechanical and the chemical equilibriums at $T^{*}=0.855$ (c) and $0.7(\mathrm{~d})$. In the positive pressure case with $T^{*}=0.855$, the paths and $\Delta G \mathrm{~s}$ in the two equilibriums are close each other, whereas the deviations in them are large in the negative pressure case. Panel (d) also shows the path of bubble growth across the critical size by the doted line.

tension from the bulk value for the nanosized critical bubbles. In Sec. III, we adopt the Tolman correction for the surface tension [26] and fix the model parameter, the Tolman length, by using the results of MD simulations.

Before proceeding to the evaluation of the prefactor, we show some examples of $\Delta G$ in Fig. 1. For the comparison with molecular dynamics simulations in Sec. III, here we consider a Lennard-Jones liquid. Figures 1 (a) and 1(b) show $\Delta G$ as a function of the bubble radius $r$ for the temperatures $T^{*}$ (三 $k T / \varepsilon)=0.855$ and 0.7 , respectively, where $\varepsilon$ is the binding energy of the Lennard-Jones potential. At each temperature, the liquid pressures are set to be $0.017 \varepsilon \sigma^{-3}$ and $-0.16 \varepsilon \sigma^{-3}$, respectively, where $\sigma$ is the unit length in the Lennard-Jones potential. The equilibrium pressures are given by $0.046 \varepsilon \sigma^{-3}$ and $0.010 \varepsilon \sigma^{-3}$ and the surface tensions are $0.089 \varepsilon \sigma^{-2}$ and $0.33 \varepsilon \sigma^{-2}$ respectively at each temperature [26]. Here, we set $v_{\mathrm{l}} / v_{\mathrm{g}}=0$. To evaluate $\Delta G$, we also fix $i$ (or $P_{\mathrm{g}}$ ). In panels (a) and (b), we plot $\Delta G$ for bubbles in mechanical equilibrium (solid lines) and in chemical equilibrium (dashed lines). The thin solid lines show $\Delta G$ for various constant bubble molecule numbers $i$. Panels (c) and (d) show the relation of $i$ and $r$ for the mechanical and the chemical equilibriums at $T^{*}=0.855$ and 0.7 .

In the positive pressure case of panel (a), both $\Delta G$ in the mechanical and the chemical equilibria are similar to one another. They agree exactly at their maxima-corresponding to the critical size. At other radii, $\Delta G$ is slightly smaller at chemical equilibrium. The minima of each constant- $i$ line are located on the line of the mechanical equilibrium. Thus we find that the critical size corresponds to the saddle point.

In the negative pressure case of panels (b) and (d), the mechanical equilibrium lines deviate considerably from those in chemical equilibrium. The maximum of $\Delta G$ in the chemical equilibrium corresponds to the minimum of the mechanical equilibrium case. This also shows that it is the saddle point. Panel (d) shows that the number of molecules of growing critical bubbles increases for the chemical equilibrium case whereas it decreases for the mechanical equilibrium case. These facts indicate that chemical equilibrium is more realistic than mechanical equilibrium. In the next subsection, we examine the growth of bubbles using the model of Kagan [41] to obtain the prefactor. It also gives us the path around the critical size. In panel (d), we also plot the obtained path across the critical size (dotted line), which is close to the line of the chemical equilibrium.

\section{Nucleation rate prefactor}

Compared to droplet nucleation, bubble nucleation requires a more detailed treatment due to the bubble compressibility. The vapor pressure in the bubbles varies as they grow and significantly affects their growth rates and the pre-exponential factor in the CNT expression for the nucleation rate, as well as the bubble formation energy. Kagan [41] solved the two-dimensional bubble evolution and showed that the pre-exponential factor is strongly dependent on the ambient liquid pressure. Based on the method of Kagan [41], we evaluate the exact prefactor $J_{0}$.

The nucleation rate is usually given by

$$
J=\frac{1}{\int[D n(i)]^{-1} d i} \simeq Z D_{c} n_{e}\left(i_{c}\right),
$$

where the Zeldovich factor $Z$ is

$$
Z=\left[-\frac{1}{2 \pi k T}\left(\frac{d^{2} \Delta G}{d i^{2}}\right)_{i_{c}}\right]^{1 / 2}
$$

and $D$ is the diffusion coefficient in the $i$ space given by [41]

$$
D=-k T\left(\frac{d i}{d t}\right) /\left(\frac{d \Delta G}{d i}\right)
$$

and $D_{c}=D\left(i_{c}\right)$. Note that $Z$ and $D_{c}$ are evaluated at the critical size $i_{\mathrm{c}}$ (or $r_{\mathrm{c}}$ ). Since the denominator and fraction in Eq. (31) both vanish at the critical size, we evaluate it with the second derivatives

$$
D_{\mathrm{c}}=-k T \frac{d}{d i}\left(\frac{d i}{d t}\right)_{i_{c}} /\left(\frac{d^{2} \Delta G}{d i^{2}}\right)_{i_{c}} .
$$

From Eq. (29), the prefactor in the nucleation rate is $J_{0}=$ $Z D_{c}$. We use $r$ instead of $i$ in the equations for convenience, and rewrite $J_{0}$ as

$$
J_{0}=\left(\frac{k T}{2 \pi} /\left|\frac{d^{2} \Delta G}{d r^{2}}\right|_{r_{c}}\right)^{1 / 2} A\left(\frac{d i}{d r}\right)_{i_{c}},
$$


where

$$
A=\left[\frac{d}{d r}\left(\frac{d r}{d t}\right)\right]_{r_{c}} .
$$

The growth rate of a spherical bubble is described by the Rayleigh-Plesset equation [28],

$$
r \frac{d^{2} r}{d t^{2}}=-\frac{3}{2}\left(\frac{d r}{d t}\right)^{2}+\frac{v_{l}}{m}\left(P_{g}-P_{l}-\frac{2 \gamma}{r}-\frac{4 \eta}{r} \frac{d r}{d t}\right),
$$

where $\eta$ is the viscosity of the liquid. The evaporation rate from the bubble surface, i.e., the time evolution of the molecule number in a bubble $i$, is given by $[1,41]$

$$
\frac{d i}{d t}=4 \pi r^{2} \frac{\alpha}{1+\epsilon} \frac{P_{\mathrm{eq}}-P_{\mathrm{g}}}{\sqrt{2 \pi m k T}},
$$

where $\alpha$ is the evaporation coefficient (often taken to be unity). The factor $\epsilon$ is introduced to include the effect of the temperature difference between the ambient liquid temperature $T$ and that at the bubble surface [41] which is given by $\epsilon=$ $\alpha d q r_{\mathrm{c}} /(\lambda \sqrt{2 \pi m k T})$, where $q$ is the latent heat of evaporation per molecule, $\lambda$ is the thermal conductivity coefficient of the liquid, and $d=d P_{\mathrm{eq}} / d T$.

Bubbles smaller than the critical size shrink, while larger ones grow. At the critical size, the growth rate therefore vanishes. So we can put $d r / d t$ around $r \simeq r_{\mathrm{c}}$ as

$$
\frac{d r}{d t}=A\left(r-r_{c}\right)
$$

Since the vapor pressure in the critical bubble is approximately equal to $P_{\text {eq }}$, the vapor pressure is given by

$$
P_{\mathrm{g}}=P_{\mathrm{eq}}+\frac{d P_{\mathrm{g}}}{d r}\left(r-r_{c}\right)
$$

around $r \simeq r_{\mathrm{c}}$, where we have neglected the small terms proportional to $v_{\mathrm{l}} / v_{\mathrm{g}}$.

Substituting Eq. (38) into Eq. (36) and transforming the left hand side of Eq. (36) by the use of Eqs. (7), (37), and (38), we obtain

$$
\frac{d P_{\mathrm{g}}}{d r}=-\frac{1+\epsilon}{\alpha V_{\mathrm{th}}} P_{\mathrm{eq}} A\left(1+\frac{1+\epsilon}{3 \alpha V_{\mathrm{th}}} r_{\mathrm{c}} A\right)^{-1},
$$

where $V_{\text {th }}$ is the thermal velocity defined by $\sqrt{k T /(2 \pi m)}$. Substituting Eqs. (37)-(39) into Eq. (35), we obtain the equation for $A$ :

$$
\begin{aligned}
A^{2} & +\frac{1}{\rho_{\mathrm{l}} r_{\mathrm{c}}}\left[\frac{1+\epsilon}{\alpha V_{\mathrm{th}}} P_{\mathrm{eq}}\left(1+\frac{1+\epsilon}{3 \alpha V_{\mathrm{th}}} r_{\mathrm{c}} A\right)^{-1}+\frac{4 \eta}{r_{\mathrm{c}}}\right] \\
\times & A-\frac{2 \gamma}{\rho_{\mathrm{l}} r_{\mathrm{c}}^{3}}=0 .
\end{aligned}
$$

Equation (40) corresponds to the cubic equation derived by Kagan [41].

Here we present a simple approximate solution to $A$. Since the bubble growth rate is significantly slower than the thermal velocity $V_{\text {th }}$, we assume that $A \ll 3 \alpha V_{\text {th }} / r_{\mathrm{c}} /(1+\epsilon)$. Then, the factor in Eq. (40) is rewritten as

$$
\left(1+\frac{1+\epsilon}{3 \alpha V_{\mathrm{th}}} r_{\mathrm{c}} A\right)^{-1} \simeq 1-\frac{1+\epsilon}{3 \alpha V_{\mathrm{th}}} r_{\mathrm{c}} A
$$

which reduces Eq. (40) to a quadratic equation and we obtain an approximate solution of $A$ as

$$
A=A_{\text {ine }}\left\{\sqrt{\left(\frac{A_{\text {ine }}}{2 A_{\text {eva }}}+\frac{A_{\text {ine }}}{2 A_{\text {vis }}}\right)^{2}+1}-\frac{A_{\text {ine }}}{2 A_{\text {eva }}}-\frac{A_{\text {ine }}}{2 A_{\text {vis }}}\right\} .
$$

The factors in Eq. (42) $A_{\text {eva }}, A_{\text {vis }}$, and $A_{\text {ine }}$ are determined by the evaporation, the inertia of the fluid motion, and the viscosity at the surface region of the bubble, respectively, and given by

$$
\begin{gathered}
A_{\mathrm{eva}}=\frac{2 \gamma}{r_{\mathrm{c}}^{2}} \frac{\alpha V_{\mathrm{th}}}{(1+\epsilon) P_{\mathrm{eq}}}, \\
A_{\mathrm{vis}}=\frac{\gamma}{2 r_{\mathrm{c}} \eta}, \\
A_{\text {ine }}=\left(\frac{2 \gamma}{\rho_{\mathrm{l}} r_{\mathrm{c}}^{3} a}\right)^{1 / 2},
\end{gathered}
$$

with

$$
a=1-\frac{(1+\epsilon)^{2} P_{\mathrm{eq}}}{3 \alpha^{2} V_{\mathrm{th}}^{2} \rho_{\mathrm{l}}} .
$$

In the limit $A_{\text {eva }} \ll A_{\text {vis }}, A_{\text {ine }}$, the growth is regulated by the evaporation process and $A=A_{\text {evap. }}$. If $A_{\text {ine }}\left(A_{\text {vis }}\right)$ is the smallest of them, the growth is determined by the inertia (the viscosity) of the fluid and $A$ is given by $A_{\text {ine }}\left(A_{\text {vis }}\right)$. We recall that this approximate solution of Eq. (42) is valid in the case where $A \ll 3 \alpha V_{\text {th }} / r_{c} /(1+\epsilon)$.

To evaluate $J_{0}$ of Eq. (33), we also need $d^{2} \Delta G / d i^{2}$ and $d i / d r$. Using Eq. (39), we obtain

$$
\begin{aligned}
\frac{d^{2} \Delta G}{d r^{2}} & =\left(\frac{\partial^{2} \Delta G}{\partial r^{2}}\right)_{P_{\mathrm{g}}}+\left(\frac{\partial^{2} \Delta G}{\partial P_{\mathrm{g}}^{2}}\right)_{r}\left(\frac{d P_{\mathrm{g}}}{d r}\right)^{2} \\
& =-8 \pi \gamma\left[1-\frac{3 P_{\mathrm{eq}}}{P_{\mathrm{eq}}-P_{1}}\left(1+\frac{3 P_{\mathrm{eq}}}{P_{\mathrm{eq}}-P_{1}} \frac{A_{\mathrm{eva}}}{A}\right)^{-2}\right],
\end{aligned}
$$

and

$$
\frac{d i}{d r}=4 \pi r_{\mathrm{c}}^{2} \frac{P_{\mathrm{eq}}}{k T} /\left(1+\frac{P_{\mathrm{eq}}-P_{1}}{3 P_{\mathrm{eq}}} \frac{A}{A_{\mathrm{eva}}}\right) .
$$

Their $P_{\text {eq }}$ dependencies affect $J_{0}$. Thus by the use of $A$, we find the expression for the prefactor in the nucleation rate:

$$
\begin{aligned}
J_{0}= & \frac{\alpha}{1+\epsilon} \sqrt{\frac{2 \gamma}{\pi m}} \frac{\frac{A}{A_{\mathrm{eva}}}}{1+\frac{P_{\mathrm{eq}}-P_{1}}{3 P_{\mathrm{eq}}} \frac{A}{A_{\mathrm{eva}}}} \\
& \times\left[1-\frac{3 P_{\mathrm{eq}}}{P_{\mathrm{eq}}-P_{1}}\left(1+\frac{3 P_{\mathrm{eq}}}{P_{\mathrm{eq}}-P_{1}} \frac{A_{\mathrm{eva}}}{A}\right)^{-2}\right]^{-1 / 2} .
\end{aligned}
$$

Note that the explicit expression of the exact prefactor was not presented by Kagan [41].

Figure 2 shows the prefactor given by Eq. (49) as a function of the liquid pressure $P_{1}$ for Lennard-Jones liquid with $T^{*}=$ $0.6,0.7$, and 0.855 . The equilibrium pressure and the surface energy are given by $P_{\mathrm{eq}}=0.0034 \varepsilon \sigma^{-3}$ and $\gamma=0.51 \varepsilon \sigma^{-2}$, respectively at $T^{*}=0.6$ [26]. Here, the evaporation coefficient 

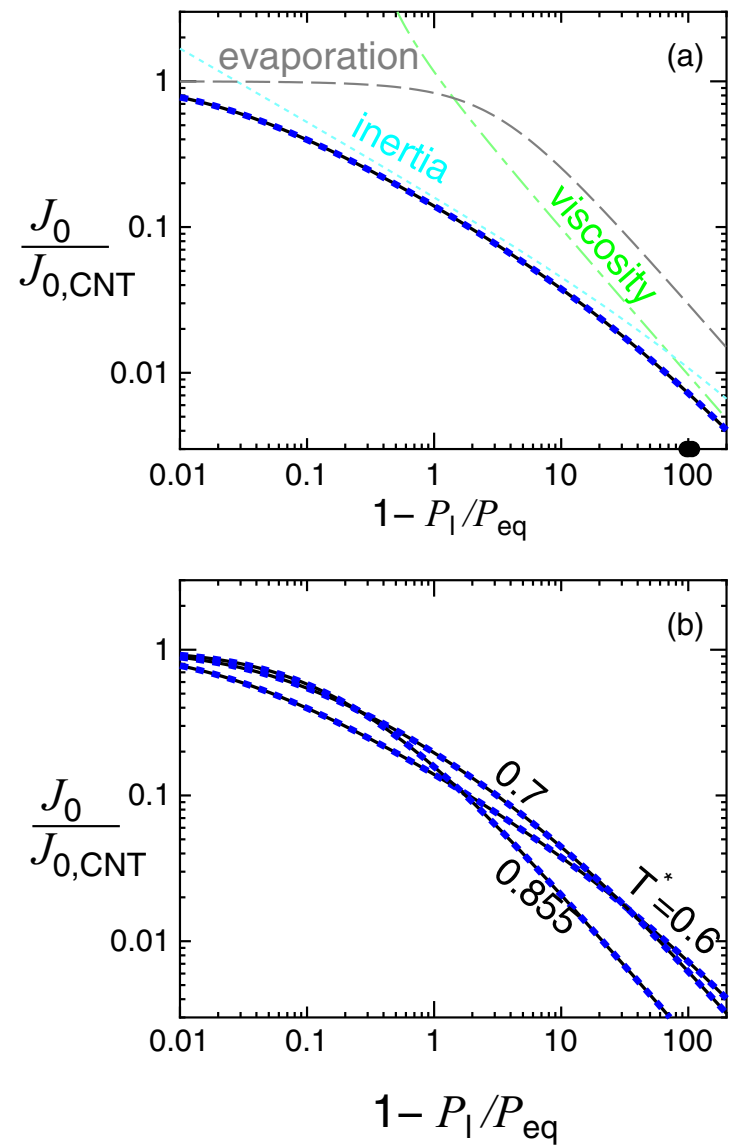

FIG. 2. (Color online) The prefactor obtained by Eq. (49) as a function of the liquid pressure $P_{1}$. Panel (a) shows the case of $T^{*}=0.6$ (see text for details). The solid line presents the prefactor with the exact solution of $A$ to Eq. (40) whereas the dashed line uses the approximate solution of Eq. (42). Equation (42) reproduced very well the exact solution within the accuracy of $0.1 \%$. The prefactors calculated with $A=A_{\text {eva }}, A_{\text {ine }}$, or $A_{\text {vis }}$ are also plotted with gray dashed, light blue dotted, or green dotted-dashed curves. The liquid pressures used in the molecular dynamics simulations (see Sec. III) are plotted by circles on the horizontal axis. The prefactors for various temperatures $T^{*}=0.6,0.7$, and 0.855 are shown in panel (b).

$\alpha$ is set to unity and $\epsilon$ to zero. The viscosity $\eta$ is set to be $0.6 \varepsilon \tau \sigma^{-3}$ with the time unit $\tau$ according to Angélil et al. [28].

When the liquid pressure is very close to the equilibrium value, $J_{0}$ agrees with the CNT value of Eq. (4). In this case, the bubble growth is regulated by the evaporation, and the mechanical equilibrium is almost satisfied on the path of bubble growth near the critical size. For a large negative pressure, on the other hand, the prefactor can be significantly smaller than the CNT value by a factor $\sim 10^{3}$ because of the viscosity and inertia effects in the liquid. In this case, chemical equilibrium holds rather than the mechanical one on the growing path. We obtain this growing path near the critical size in the $r-i$ plane, by evaluating $d i / d r$ with Eq. (48). The obtained path is plotted in Fig. 1(d).

\section{COMPARISON BETWEEN THE CLASSICAL NUCLEATION RATE AND THE EXACT FORMULA}

We first compare the theoretical nucleation models described in Sec. II with the molecular dynamics simulations

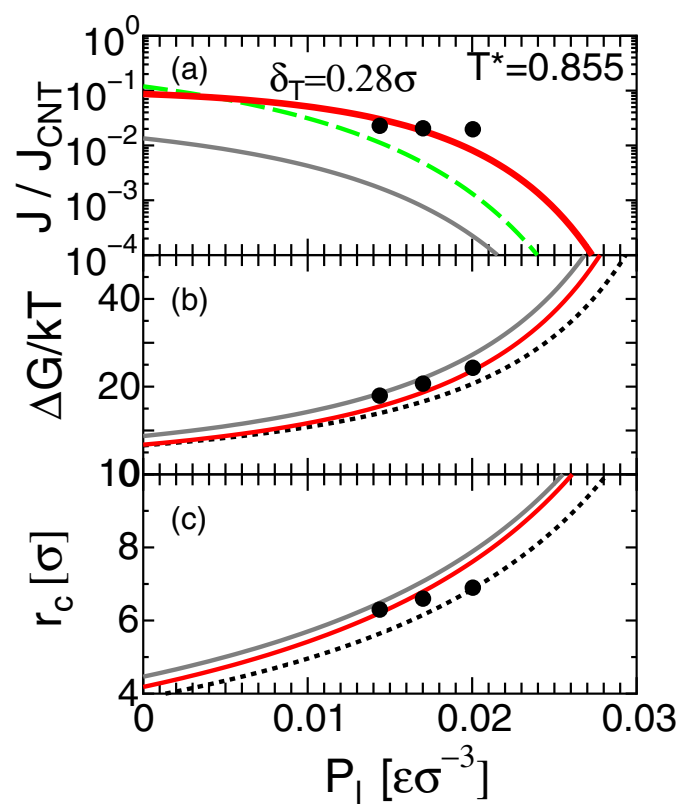

FIG. 3. (Color online) Comparison between the improved formula and the CNT for (TSF) Lennard-Jones fluid at $T^{*}=0.855$. Panel (a) shows the comparison in $J$. The improved nucleation rate given by Eqs. (28) and (49) without the Tolman correction is plotted with the gray line. The improved formula with the Tolman correction is the thick red curve, where $\delta_{T}$ is set to be $0.28 \sigma$. The nucleation rate given by Eq. (28) with $J_{0}=J_{0, \mathrm{CNT}}$ is the green dashed curve. Panel (b) shows the free energy for formation of a critical bubble for the PCNT without and with the Tolman correction (gray and red solid curves) and for the CNT (dotted curve). Panel (c) shows the critical bubble radii (same models and line styles as above). In all panels, the results obtained by the molecular dynamics simulations [26] are plotted with filled circles.

by [26]. The MD simulations used molecules with a truncated force-shifted (TSF) Lennard-Jones potential and a cutoff length of $2.5 \sigma$. In the models, we thus use the values of TSF Lennard-Jones liquid for the thermodynamics data such as the surface tension and the equilibrium pressure. The evaporation coefficient $\alpha$ is assumed to be unity. In the calculations of the improved nucleation rate, we use Eq. (28). In Fig. 3, we show comparisons at $T^{*}=0.855$. We plot the results of MD simulations (with filled circles) and four theoretical models. The first model is the CNT. The CNT nucleation rate is given by Eq. (5). Other nucleation rates are normalized with respect to this in Fig. 3(a). The second (green dashed curve) is the PCNT nucleation rate which is given by Eq. (28) with $J_{0}=J_{0 \text {, CNT }}$ of Eq. (4). The Poynting correction factor is evaluated from Eqs. (24) and (26) to include the nonideal gas effect.

The third (gray curve) is our improved expression given by Eqs. (28) and (49). The last one (red curve) uses the same improved expression as the third one, but also includes the Tolman correction to the surface tension. The Tolman correction describes the size-dependent surface tension as

$$
\gamma=\gamma_{\infty} /\left(1+2 \delta_{T} / r\right)
$$

where $\gamma_{\infty}$ is the surface energy of the planar interface. The Tolman length in the Tolman correction $\delta_{T}$ expresses the curvature dependence on the surface tension of the bubble. If 
$\delta_{T}$ is positive, the surface tension of small bubbles or droplets is smaller than the planar one.

In Fig. 3(a), we find that the improved formula (gray curve) can be smaller than the CNT (the PCNT) by a factor of $\sim 10^{-4}(\sim 1 / 5)$. Figure 3 shows that the Tolman correction is necessary to reproduce the results of the MD simulations. From the fitting, we obtain $\delta_{T}=0.28 \sigma$. Figure 3(b) shows the peak values of the free energy for bubble formation. We find the PCNT correction almost reproduces $\Delta G\left(r_{\mathrm{c}}\right)$ in the MD simulations. Figure 3(c) shows the critical bubbles $r_{c}$ obtained from the theoretical models and MD simulations. All models successfully reproduce the $r_{c}$ measured in the MD simulations. Note that the Tolman length of $0.28 \sigma$ obtained by fitting is much smaller than the critical radius. Thus the correction to the surface tension of Eq. (50) contributes only at the $10 \%$ level, yet significantly affects the nucleation rate due to the high sensitivity that the surface tension has on $J$ [see Eq. (28)].

Figure 4 shows the comparisons in $J$ at $T^{*}=0.6,0.7$, and 0.8 , where the equilibrium pressure and the surface energy are given by $P_{\mathrm{eq}}=0.0303 \varepsilon \sigma^{-3}$ and $\gamma=0.17 \varepsilon \sigma^{-2}$, respectively, at $T^{*}=0.8$ [26]. We find that deviations in the improved formula (gray curves) from the CNT can be several orders of magnitude in all cases. Compared with the values obtained by MD simulations, the improved formula without the Tolman correction gives significantly lower nucleation rates. By the fitting, we obtain $\delta_{T}=0.29 \sigma, 0.32 \sigma$, and $0.38 \sigma$ for $T^{*}=$ $0.8,0.7$, and 0.6 , respectively. The fits at $T^{*}=0.6-0.855$ show that the Tolman length depends weakly on temperature. Since the radii of the critical bubbles are $3-7 \sigma$ in the MD simulations, we see from Eq. (50) that the obtained Tolman

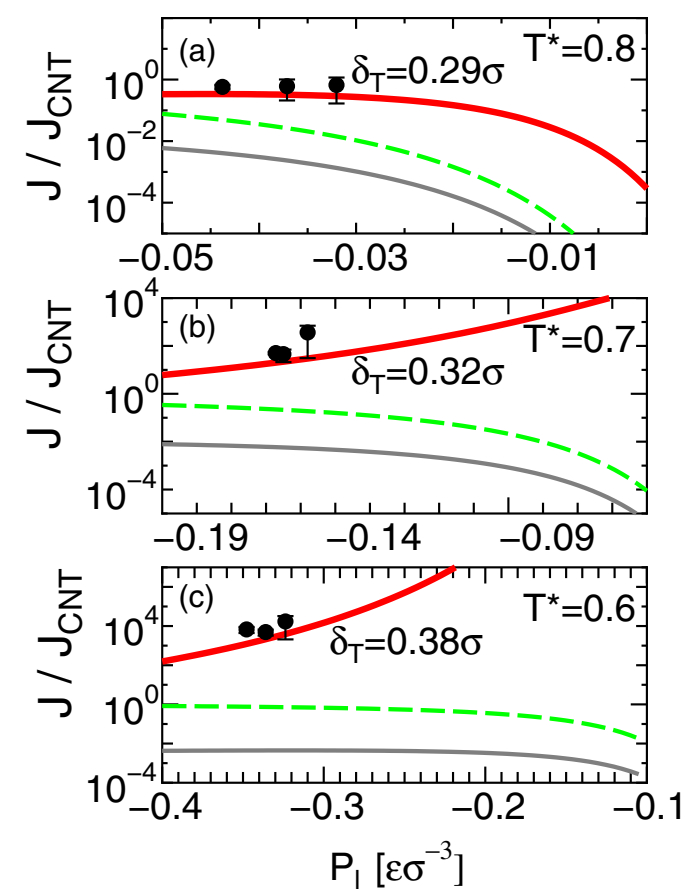

FIG. 4. (Color online) The same as Fig. 3(a), but for $T^{*}=0.8$, 0.7 , and 0.6. In the improved formula with the Tolman correction (red curves), the Tolman length $\delta_{T}$ are set to be $\delta_{T}=0.29,0.32$, and $0.38 \sigma$ for $T^{*}=0.8,0.7$, and 0.6 , respectively.
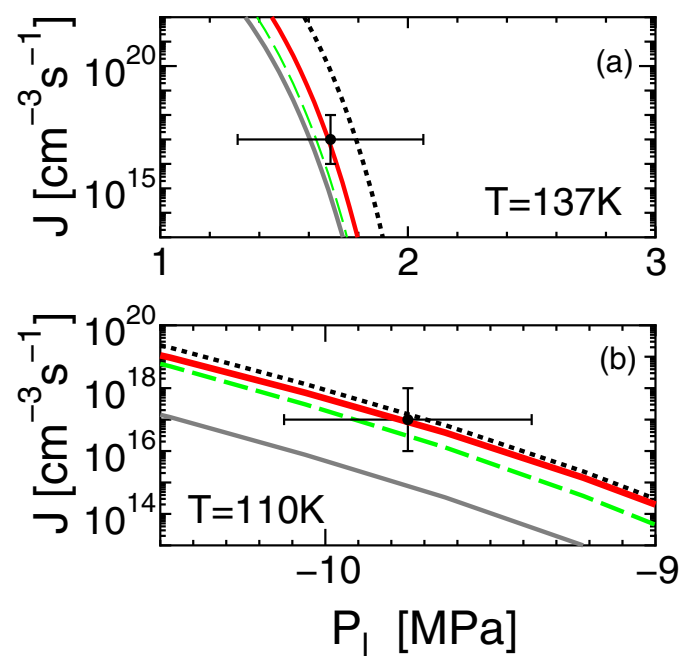

FIG. 5. (Color online) Comparison between the theoretical models and laboratory experiments in $J$ for argon at $T=137 \mathrm{~K}$ (a) and $110 \mathrm{~K}(\mathrm{~b})$. The improved nucleation rates without the Tolman correction and with the Tolman correction of $\delta_{T}=0.3 \sigma$ at $T=137 \mathrm{~K}$ $\left(\delta_{T}=0.13 \sigma\right.$ at $\left.T=110 \mathrm{~K}\right)$ are shown by the gray and red solid curves, respectively. The nucleation rate given by Eq. (28) with $J_{0}=J_{0, \text { CNT }}$ is shown by the green dashed curve. The CNT is shown by the dotted curve. In the thermodynamics data such as the surface tension and the equilibrium pressure, we use the data of argon [49]. We also show the experimental results [12] (circle), where the error in the liquid pressure is determined from the two points at $T=137 \mathrm{~K}$ in [12].

lengths of $\simeq 0.3 \sigma$ correspond to corrections of $10-20 \%$ to the surface tension from the bulk values. As seen in Figs. 3 and 4, these small corrections to the surface tension actually improve much the predictions of the nucleation-rate formula at $T^{*}=0.6-0.855$.

We also make a comparison with the argon bubble nucleation experiments by [12]. Figure 5 shows the comparisons in $J$ at $T=137$ and $110 \mathrm{~K}$. As for the thermodynamics data in models such as the surface tension and the equilibrium pressure, we use the data of argon [49]. We find the deviations between the CNT and the improved formula can be several order of magnitudes in these cases, too.

The experimental result is consistent with all of the theoretical predictions within the error at $T=137 \mathrm{~K}$. Thus it is difficult to fix the Tolman length in this case. For $T=110 \mathrm{~K}$, the improved formula gives significantly smaller nucleation rates than the experimental result. The fitting with the Tolman correction indicates that the Tolman length $\delta_{T}$ is $0.13 \sigma \pm 0.14 \sigma$. The error in $\delta_{T}$ comes from the errors in $J$ and $P_{l}$ in the laboratory experiment. This value of the Tolman length can be marginally consistent with the ones from the fits to the MD simulations. To further constrain $\delta_{T}$ more experimental data are needed.

In Fig. 6, we plot the Tolman length for bubbles obtained from our analysis (red circles) as a function of $T^{*}$ as well as those in the previous studies (other red symbols). As for our fitting data with the argon experiment in Fig. 5(b) (red open circle), the normalized temperature $T^{*}$ is obtained as 0.92 , because $\varepsilon / k=120 \mathrm{~K}$ for argon. Our results indicate that the 


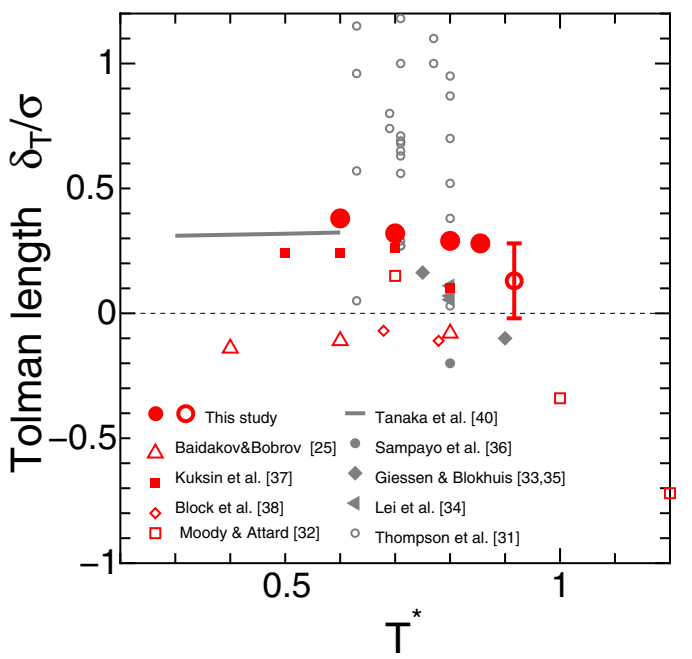

FIG. 6. (Color online) The Tolman length for bubbles as a function of temperature obtained by this study and the previous ones $[25,32,37,38]$ (red symbols). Our analysis with MD simulations (filled circles) and the experiment at $110 \mathrm{~K}$ (open circle) suggest the Tolman length is approximately given by $\delta_{T}=0.3 \sigma$. For reference, the Tolman length for droplets are also shown with gray symbols $[31,33-36,40]$.

Tolman length is almost constant with temperature. Our results agree quite well with the previous results of [37].

Baidakov and Bobrov [25] and Block [38] obtained small negative values in their molecular dynamics (MD) and Monte Carlo (MC) simulations, respectively. The deviation in the Tolman length between their results and ours comes from the different definitions of $\delta_{T}$ (or the different curvature dependence of the surface tension).

Their obtained surface tensions are always less than the bulk values, which is consistent with our curvature dependence with a positive Tolman length.

For example, Baidakov and Bobrov [25] gave the curvature dependence of the surface tension as

$$
\gamma=\gamma_{\infty} /\left(1+2 \delta_{T} / r+l^{2} / r^{2}\right)
$$

If Eq. (50) is used for the evaluation of $\delta_{T}$ instead of their curvature dependence, their results give positive $\delta_{T}$. In fact Eq. (50) with $\delta_{T}=0.3 \sigma$ also very successfully reproduces the surface tension measurements by Baidakov and Bobrov [25] (in their Fig. 9). This means that the results by Baidakov and Bobrov also agree well with ours.

Moody and Attard [32] also obtained negative Tolman lengths at $T^{*} \geqslant 1$ from their MC simulations. Even at the high temperatures, nevertheless, their obtained surface tension increases with the bubble radius at $r \lesssim 2 \sigma$ (see their Figs. 10 and 11), which is also consistent with our curvature dependence.

Recent calculations with density functional theory [50,51] also result in negative Tolman lengths, by using a different curvature dependence similar to [25]. However, they also obtained smaller surface tensions than the bulk and, in this sense, their results are consistent with ours.

For reference, we plot the Tolman length for droplets in Fig. 6. We find the value for droplets by [40] is consistent with our results for bubbles, although there is some scatter in the previous values. Our results suggest that the Tolman length is approximately given by $\simeq 0.3 \sigma$, and is temperature independent. The Tolman correction with $\delta_{T}=0.3 \sigma$ significantly improves the prediction of the nucleation rate with our nucleation rate model.

\section{CONCLUSION}

We have revised the expression of the bubble nucleation rate based on the classical theory. In bubble nucleation, the prefactor is far more complex than in droplet nucleation because bubble growth is regulated by many processes (i.e., evaporation, thermal conduction, viscosity, and inertia of liquid). This difference in the prefactor between the bubble and droplet cases has been overlooked in many studies. We have also compared the improved expression of the nucleation rate with results of the MD simulations and laboratory experiments. Our findings are summarized below.

(i) In bubble nucleation, the prefactor is strongly dependent on the degree of nonequilibrium, i.e., the liquid pressure, whereas it is constant in the droplet case. In the case of a large negative liquid pressure (or the highly viscous case), the prefactor can be far smaller than the droplet case by a factor of $10^{-3}-10^{-1}$ (Fig. 2).

(ii) When the liquid pressure is slightly below the equilibrium pressure, the deviations in the free energy for bubble formation from the CNT become large. For example, in dark matter detection experiments which use superheated liquids as targets [6-9], the degree of nonequilibrium is very small. For such a near-equilibrium case, the PCNT should be used instead of CNT.

(iii) Comparisons of our improved expression for the nucleation rates $J$ to results from MD simulations and laboratory experiments suggest that the surface tension depends on the bubble size at the nanoscale level. The improved expression including the Tolman correction to the surface tension with a small Tolman length of $\simeq 0.3 \sigma$ leads to good agreements with the recent MD simulations and laboratory experiments (Fig. 6).

\section{ACKNOWLEDGMENTS}

We thank two anonymous reviewers for valuable suggestions. This work was supported in part by JSPS KAKENHI Grants No. 26108503, No. 2540054, No. 15H05731, and No. 26287101. J.D. and R.A. were supported by the Swiss National Science Foundation (SNF).
[1] M. Blander and J. L. Katz, AIChE J. 21, 833 (1975).
[2] Y. Iida and K. Okuyama, Int. J. Heat Mass Transfer 37, 2771 (1994). 
[3] R. Sparks, J. Volcanol. Geotherm. Res. 3, 1 (1978).

[4] A. Toramaru, J. Geophys. Res. 100, 1913 (1995).

[5] K. Yamada, H. Tanaka, K. Nakazawa, and H. Emori, J. Geophys. Res. 110, B02203 (2005).

[6] E. Behnke, J. I. Collar, P. S. Cooper, K. Crum, M. Crisler, M. Hu, I. Levine, D. Nakazawa, H. Nguyen, B. Odom et al., Science 319, 933 (2008).

[7] S. Archambault, F. Aubin, M. Auger, M. Beleshi, E. Behnke, J. Behnke, B. Beltran, K. Clark, X. Dai, M. Das et al. (The PICASSO Collaboration), New J. Phys. 13, 043006 (2011).

[8] M. Felizardo, T. A. Girard, T. Morlat, A. C. Fernandes, A. R. Ramos, J. G. Marques, A. Kling, J. Puibasset, M. Auguste, D. Boyer et al., Phys. Rev. Lett. 108, 201302 (2012).

[9] E. Behnke, T. Benjamin, S. J. Brice, D. Broemmelsiek, J. I. Collar, P. S. Cooper, M. Crisler, C. E. Dahl, D. Fustin, J. Hall et al., Phys. Rev. D 88, 021101 (2013).

[10] C. C. Coussios and R. A. Roy, Annu. Rev. Fluid Mech. 40, 395 (2008).

[11] J.-E. Blatteau, J. B. Souraud, E. Gempp, and A. Boussuges, Aviat. Space Environ. Med. 77, 1068 (2006).

[12] V. E. Vinogradov, P. A. Pavlov, and V. G. Baidakov, J. Chem. Phys. 128, 234508 (2008).

[13] K. K. Tanaka, H. Tanaka, K. Kawamura, and K. Nakazawa, J. Chem. Phys. 122, 184514 (2005).

[14] B. R. Novak, E. J. Maginn, and M. J. McCready, Phys. Rev. B 75, 085413 (2007).

[15] M. Sekine, K. Yasuoka, T. Kinjo, and M. Matsumoto, Fluid Dyn. Res. 40, 597 (2008).

[16] S.-i. Tsuda, S. Takagi, and Y. Matsumoto, Fluid Dyn. Res. 40, 606 (2008).

[17] Z.-J. Wang, C. Valeriani, and D. Frenkel, J. Phys. Chem. B 113, 3776 (2009).

[18] H. Watanabe, M. Suzuki, and N. Ito, Phys. Rev. E 82, 051604 (2010).

[19] K. K. Tanaka, H. Tanaka, T. Yamamoto, and K. Kawamura, J. Chem. Phys. 134, 204313 (2011).

[20] S. L. Meadley and F. A. Escobedo, J. Chem. Phys. 137, 074109 (2012).

[21] J. L. F. Abascal, M. A. Gonzalez, J. L. Aragones, and C. Valeriani, J. Chem. Phys. 138, 084508 (2013).

[22] H. Watanabe, M. Suzuki, and N. Ito, Comput. Phys. Commun. 184, 2775 (2013).

[23] J. Diemand, R. Angélil, K. K. Tanaka, and H. Tanaka, J. Chem. Phys. 139, 074309 (2013).

[24] K. K. Tanaka, A. Kawano, and H. Tanaka, J. Chem. Phys. 140, 114302 (2014).

[25] V. G. Baidakov and K. S. Bobrov, J. Chem. Phys. 140, 184506 (2014).
[26] J. Diemand, R. Angélil, K. K. Tanaka, and H. Tanaka, Phys. Rev. E 90, 052407 (2014).

[27] R. Angélil, J. Diemand, K. K. Tanaka, and H. Tanaka, J. Chem. Phys. 140, 074303 (2014).

[28] R. Angélil, J. Diemand, K. K. Tanaka, and H. Tanaka, Phys. Rev. E 90, 063301 (2014).

[29] V. E. Vinogradov, P. A. Pavlov, and V. G. Baidakov, Chem. Phys. Lett. 474, 294 (2009).

[30] R. C. Tolman, J. Chem. Phys. 17, 333 (1949).

[31] S. M. Thompson, K. E. Gubbins, J. P. R. B. Walton, R. A. R. Chantry, and J. S. Rowlinson, J. Chem. Phys. 81, 530 (1984).

[32] M. P. Moody and P. Attard, J. Chem. Phys. 115, 8967 (2001).

[33] A. E. van Giessen and E. M. Blokhuis, J. Chem. Phys. 116, 302 (2002).

[34] Y. A. Lei, T. Bykov, S. Yoo, and X. C. Zeng, J. Am. Chem. Soc. 127, 15346 (2005).

[35] A. E. van Giessen and E. M. Blokhuis, J. Chem. Phys. 131, 164705 (2009).

[36] J. G. Sampayo, A. Malijevský, E. A. Müller, E. de Miguel, and G. Jackson, J. Chem. Phys. 132, 141101 (2010).

[37] A. Yu. Kuksin, G. E. Norman, V. V. Pisarev, V. V. Stegailov, and A. V. Yanilkin, Phys. Rev. B 82, 174101 (2010).

[38] B. J. Block, S. K. Das, M. Oettel, P. Virnau, and K. Binder, J. Chem. Phys. 133, 154702 (2010).

[39] M. Horsch, H. Hasse, A. K. Shchekin, A. Agarwal, S. Eckelsbach, J. Vrabec, E. A. Muller, and G. Jackson, Phys. Rev. E 85, 031605 (2012).

[40] K. K. Tanaka, J. Diemand, R. Angélil, and H. Tanaka, J. Chem. Phys. 140, 194310 (2014).

[41] Y. Kagan, Russ. J. Phys. Chem. 34, 42 (1960).

[42] L. Gunther, Am. J. Phys. 71, 351 (2003).

[43] J. W. P. Schmelzer, G. Sh. Boltachev, and V. G. Baidakov, J. Chem. Phys. 124, 194503 (2006).

[44] M. J. Uline and D. S. Corti, Phys. Rev. Lett. 99, 076102 (2007).

[45] M. J. Uline, K. Tarabi, and D. S. Corti, J. Chem. Phys. 133, 174511 (2010).

[46] K. Torabi and D. S. Corti, J. Phys. Chem. B 117, 12479 (2013).

[47] D. Landau and E. M. Lifshitz, Stastistical Physics (Pergamon, Oxford, 1980).

[48] P. G. Debenedetti, Metastable liquids. Concepts and Principles (Princeton University Press, Princeton, 1996).

[49] K. Iland, J. Wölk, R. Strey, and D. Kashchiev, J. Chem. Phys. 127, 154506 (2007).

[50] E. M. Blokhuis and A. E. van Giessen, J. Phys.: Condens. Matter 25, 225003 (2013).

[51] O. Wilhelmsen, D. Bedeaux, and D. Reguera, J. Chem. Phys. 142, 064706 (2015). 